\title{
Madariaga, Salvador de, The Rise of the Spanish American \\ Empire. London, Hallis and Carter, 1947. 408 p.; The Fall of the Spanish American Empire. London, Hallis and Carter, 1947. 443
}

p.

\section{Boris Celovsky}

Volume 6, numéro 4, mars 1953

URI : https://id.erudit.org/iderudit/301564ar

DOI : https://doi.org/10.7202/301564ar

Aller au sommaire du numéro

Éditeur(s)

Institut d'histoire de l'Amérique française

ISSN

0035-2357 (imprimé)

1492-1383 (numérique)

Découvrir la revue

Citer ce compte rendu

Celovsky, B. (1953). Compte rendu de [Madariaga, Salvador de, The Rise of the Spanish American Empire. London, Hallis and Carter, 1947. 408 p.; The Fall of the Spanish American Empire. London, Hallis and Carter, 1947. 443 p.] Revue d'histoire de l'Amérique française, 6(4), 586-587.

https://doi.org/10.7202/301564ar d'utilisation que vous pouvez consulter en ligne. 
Madariaga, Salvador de, The Rise of the Spanish American Empire. London, Hallis \& Carter, 1947. 408 pages; The Fall of the Spanish American Empire. London, Hallis \& Carter, 1947. 443 pages.

L'historien espagnol en exil, après ses nombreux travaux monographiques sur l'histoire espagnole, publie deux forts volumes d'une remarquable envergure. Il essaie d'embrasser, dans un bref panorama, toute l'évolution de l'empire hispano-américain. La division en deux volumes n'est pas uniquement affaire de chronologie, comme le feraient supposer les deux titres de Madariaga. La division entend correspondre à des facteurs qui concourent presque parallèlement à l'évolution d'un empire, à son ascension et à sa chute. Peut-être y a-t-il là du nouveau dans les méthodes synthétiques de la science historique. De premier abord, telle méthode peut prêter le flane à d'assez graves inconvénients. Elle expose à une fatale répétition du même fait, de même qu'à des interprétations différentes, selon les exigences de la thèse. Reproche qui vaudrait ici pour l'histoire politique proprement dite. Mais l'histoire de Madariaga dépasse cette perspective. Les événements politiques y constituent un réseau mais à grosses mailles où trouvent largement place les facteurs sociaux, économiques, culturels, y compris les problèmes raciaux. Les uns assimilent volontiers une telle histoire à de la sociologie; d'autres, et nous sommes de ceux-là n'y voient que la formule de l'histoire intégrale. En outre, il appert que l'auteur s'est documenté à bonnes sources et qu'il a pratiquement tiré parti de toute la littérature sur le sujet. Mentionnons aussi que Madariaga a entrepris cette étude sur l'ascension et la chute de la puissance espagnole en Amérique, simplement pour on faire the indispensable background qui expliquerait la fin tragique de l'auteur du fédéralisme latino-américain, Simon Bolivar. 
Une bonne histoire de l'Empire hispano-américain intéresse non seulement un historien américain, mais aussi un historien canadien, notamment canadien-français. Les simples et nombreux renvois aux documents et à la littérature espagnole, riches de renseignements de premier ordre sur l'histoire des relations franco-espagnoles en Amérique, peuvent enrichir la connaissance et faciliter l'étude de l'histoire franco-américaine. De plus, les jugements de Madariaga, bien que de point de vue espagnol, contribuent à l'explication des difficiles problèmes anglo-franco-espagnols en Amérique. Madariaga nous ouvre aussi quelques perspectives intéressantes par la comparaison des histoires diverses des colonies espagnoles, françaises et anglaises. Il n'est pas, non plus, sans intérêt, de retracer les influences françaises dans la vie des colonies espagnoles, même dans le domaine administratif, (fait assez étrange quand on connaît la rigidité du système colonial espagnol), par exemple l'introduction des fonctions de l'intendant et d'autres fonctionnaires supérieurs par le décret royal du 26 juillet 1718. L'exposé des influences culturelles réciproques entre les deux groupes de colonies est surtout tiré de l'ouvrage de Charles Garcia, Antipathie des Français \& des Espagnols... Rouen 1638. Où l'exactitude de M. Madariaga nous apparaît le mieux, c'est dans l'explication de l'aventure Penalosa ("unscrupulous blackguard, as shown in his lamentable governorship of New Biscay" 2: 150) - La Salle. Pour l'auteur cette aventure n'eut d'autre résultat que d'amener l'autorité espagnole à prêter la plus grande attention à la côte de Vera Cruz et de la Floride. En somme, l'œuvre de Madariaga est un témoignage d'honnêteté et d'un notable labeur; elle apporte du nouveau, et en tant que telle, elle devient une contribution valable à l'histoire des deux Amériques.

Boris Celovsky, Heidelberg. 